\title{
KNOWLEDGE OF ANTIBIOTIC PRESCRIPTION FOR ENDODONTIC TREATMENT IN FINAL YEAR STUDENTS AND HOUSE OFFICERS.
}

\author{
Zehra Azhar Jawa ${ }^{1}$, Laila Azhar Jawa ${ }^{2}$, Jamil BDS $^{3}$, Mustafa Sajid $^{4}$, Mohsin Javed $^{5}$ \\ 1,2,5 Demonstrator Multan Medical and Dental College Multan \\ ${ }^{3}$ Associate Professor Multan Medical and Dental College Multan \\ ${ }^{4}$ Assisstant Professor Multan Medical and Dental College Multan \\ Correspondence: Mohsin Javed, Email: mohsinjavaid734@gmail.com
}

\begin{abstract}
Background: Antibiotics have been used extensively for the management of odontogenic infections since their discovery.

Objective: The objective of the study was to assess the knowledge of the antibiotic prescription during endodontic therapy and common errors made by fourth-year students and house officers at Multan Medical and Dental College. This study conducted in August 2017.

Methods: Questionnaire was designed, regarding pattern of prescription of antibiotics by the dentists and the conditions for which they were prescribed. The self investigated questionnaire investigated dental students and house officer's knowledge of the indications for prescribing antibiotics for a number of systemic clinical signs that may be associated with a dental infection.

Results: For chronic periapical lesions and chronic periapical abscess plus sinus tracts, $58.1 \%$ and $55.8 \%$ of respondents prescribed antibiotics, respectively. The drug of choice for these cases was Amoxicillin+calvulanic acid $(52.6 \%)$ and Amoxicillin alone (47.3\%). In this study the maximum antibiotics prescribed were prophylactic for congenital heart diseases $69.7 \%$ and uncontrolled diabetes mellitus $62.8 \%$.

Conclusion: This study supports the conclusion that there is a lack of knowledge about the correct indication, type, and dosage of antibiotics in dental practice.
\end{abstract}

Keywords: Antibiotics, root canal treatment, prophylaxis

\section{Introduction}

Antibiotics have been used extensively for the management of odontogenic infections since their discovery. The advent of antibiotics resulted in a significant decline in the incidence of life-threatening infections and heralded a new era in the therapy of infectious diseases, but the enthusiasm turned out to be premature1. Over the years, microbial evolutionary responses to the selected pressure exerted by antibiotics have resulted in microbial species resistant to virtually every known antibiotic agent. Over use and misuse of antibiotics has been considered the major cause for the emergence of multidrug-resistant strains2, 3 and 4. Antibiotic resistance among obligatory anaerobic bacteria is increasing, with resistance to penicillins, clindamycin and cephalosporin noted at community hospitals and major medical centers 5 .

The risk/benefit ratio should be always weighed before prescribing antibiotics. Appropriately selected patients will benefit from systemically administered antibiotics. A restricted and conservative use of antibiotics is highly recommended in endodontic practice, but indiscriminate use (including cases of pulpitis- no infection) is contrary to sound clinical practice. This may cause a selective pressure and consequent overgrowth of intrinsically resistant bacteria, predisposing patients to secondary and super infections and rendering drugs ineffective against potentially fatal medical infectious diseases 1.

Drugs are usually prescribed for a number of conditions by dentists. These drugs may cause harm to the patient if are not prescribed accordingly. Most adverse drug events $(68-75 \%)$ are reported to have been related to incorrect prescription writing6. Although these events may not be fatal, they may be a source of morbidity to the patient. The act of prescribing is becoming increasingly challenging due to multiple factors. Prescribing errors can generally be categorized into those of decision making or of prescription writing. While the former may include errors, such as under prescribing, overprescribing, irrational prescribing and inappropriate prescribing7, 8 the latter focuses on errors made while writing a prescription9. Although poor prescribing by junior doctors and students has been reported in a number of studies, partly attributing it to knowledgebased errors. 10, 11

The objective of the study was to assess the knowledge 
of the antibiotic prescription during endodontic therapy and common errors made by fourth-year students and house officers at Multan Medical and Dental College. Methodology

This is a survey based study conducted among nineteen fourth-year dental students and twenty four house officers at Multan Medical and Dental College on August 2017. A questionnaire was designed, regarding pattern of prescription of antibiotics by the dentists and the conditions for which they were prescribed. The questionnaire investigated dental students' and house officer's knowledge about the indications of antibiotics prescription for a number of systemic clinical signs that may be associated with dental infections. The clinical signs chosen were fever and malaise, evidence of systemic spread, diffused swelling, and difficulty in swallowing. The students and house officers were also asked whether some clinical conditions required antibiotics and their choice of treatment if any. These clinical conditions were acute pulpitis, acute apical abscess, chronic apical abscess with sinus tract, chronic apical periodontitis. Also a number of factors that can influence antibiotic prescriptions were investigated. The questionnaire asked whether patient's expectation of prescribing antibiotics, two session root canal treatment, one long session root canal treatment, and retreatment might be the reason for prescribing antibiotics. The next part of the questionnaire assessed knowledge on the medical conditions and dental procedures that may require prophylactic antibiotics. The dental procedures were all root canal treatments, pre and post endodontic surgeries; the medical conditions included $\mathrm{HIV}+$, hepatitis $B$, uncontrolled diabetes, congenital heart diseases, mitral valve prolapse and patients who have had prosthetic joint in the past 2 years or those with a history of cancer and radiotherapy. This questionnaire was distributed among 43 students of Multan medical and dental college. The collected data was tabulated and analyzed.

\section{Results}

The demographics of respondents are described in Table 1. Male and female dentists answered $39 \%$ and $61 \%$ of the questions respectively. Number of respondents is given in Table 2.

The drug of choice for these cases was Amoxicillin+clavulanic acid(52.6\%) and Amoxicillin alone $(47.3 \%)$. The greatest number of antibiotic prescriptions was written for acute apical abscess $(62.8 \%)$ and patient with fever and malaise $(62.7 \%)$. Overall, $11.6 \%$ of respondents always prescribed antibiotics after root canal therapy (Table 4). For chronic periapical lesions and chronic periapical abscess plus sinus tracts, $58.1 \%$ and $55.8 \%$ of respondents prescribed antibiotics, respectively. Amoxicillin+clavulanic acid was the drug of choice $(52.6 \%)$ in all correct and incorrect cases.
Table 1. Description of respondents

\begin{tabular}{|l|c|}
\hline Male & $39 \%$ \\
\hline Female & $61 \%$ \\
\hline
\end{tabular}

\section{Table 2. Number of respondents}

\begin{tabular}{|l|c|}
\hline House officers & 19 \\
\hline Final year students & 24 \\
\hline
\end{tabular}

Table 3. Antibiotics that were prescribed by respondents

\begin{tabular}{|l|c|}
\hline \multicolumn{1}{|c|}{ Antibiotic } & $\%$ \\
\hline Amoxicillin & $47.3 \%$ \\
\hline Amoxicillin + clavulanic acid & $52.6 \%$ \\
\hline Cephalosporins & $10.52 \%$ \\
\hline Clindamycin & $10.52 \%$ \\
\hline
\end{tabular}

Table 4. Conditions and percentage of antibiotic prescription

\begin{tabular}{|l|l|}
\hline \multicolumn{1}{|c|}{ Conditions } & \multicolumn{1}{|c|}{ Prescribed Antibiotics (\%) } \\
\hline Acute pulpits & $25.5 \%$ \\
\hline Acute apical Abscess & $62.8 \%$ \\
\hline Chronic apical abscess with periodontitis & $55.8 \%$ \\
\hline Chronic apical abscess with sinus tract & $58.1 \%$ \\
\hline In patient with fever and malaise & $62.7 \%$ \\
\hline In patient with diffuse swelling & $44.1 \%$ \\
\hline In patient with swelling \& difficulty in & $58.1 \%$ \\
\hline swallowing & \\
\hline In two visit root canal treatment & $13.9 \%$ \\
\hline In retreatment & $18.6 \%$ \\
\hline If patient insist & $13.9 \%$ \\
\hline In prolonged root canal treatment & $32.5 \%$ \\
\hline After root canal treatment & $11.6 \%$ \\
\hline
\end{tabular}


Table5. Conditions and percentage of Prophylactic antibiotic prescription

\begin{tabular}{|l|c|}
\hline \multicolumn{1}{|c|}{ Conditions } & Prophylactic prescription (\%) \\
\hline Always before every root canal treatment & $13.9 \%$ \\
\hline Only before endodontic surgery & $27.9 \%$ \\
\hline HIV + patients & $41.9 \%$ \\
\hline HBS + patients & $18.6 \%$ \\
\hline Uncontrolled diabetic patients & $62.8 \%$ \\
\hline Congenital heart disease (AV shunt and cardiac & $69.7 \%$ \\
\hline valve replacement) & \\
\hline Mitral valve prolapsed & $34.9 \%$ \\
\hline Prosthetic joint in past 2 years & $46.5 \%$ \\
\hline History of cancer and radiotherapy & $25.6 \%$ \\
\hline
\end{tabular}

In this study the greatest number of antibiotic prescribed were prophylactically for congenital heart diseases $69.7 \%$, and uncontrolled diabetes mellitus $62.8 \%$.

\section{Discussion}

Most oral diseases presenting are primarily inflammatory conditions that are associated with pain 1 . In a considerable number of cases dental pain is due to either acute or chronic infections of pulpal origin, which necessitates operative intervention, rather than antibiotics 1 . The vast majority of infections of endodontic origin are treated without the need for antibiotics. 1

In this study greatest numbers of antibiotic prescriptions written were for acute apical abscess $(62.8 \%)$ and patient with fever and malaise $(62.7 \%)$ which is comparable with the findings of Mohammed Reza who stated that $74 \%$ participants used antibiotics for apical abscess 12 .

Palmer et al. showed that $12.5 \%$ of British GDPs prescribed antibiotics for acute pulpitis 13 while in this study only $25.5 \%$ participants prescribed antibiotics for acute pulpitis. Antibiotics are not indicated and will not assist cases where the pulp is still vital and there are no signs of local or systemic infection/involvement14 which in contrast to this study in which most of participants used antibiotics for pulpal conditions.

The proper treatment for irreversible pulpitis cases is debridement of the root canal space. Non-surgical root canal therapy without antibiotic is usually adequate to treat irreversible pulpitis, acute pulpitis and chronic apical periodontitis and draining sinus tract. The pulpal circulation is compromised in these cases and systemic antibiotic will not reach therapeutic concentrations in the pulp. Removing the source of the infection by performing thorough non-surgical root canal therapy will usually allow healing of periradicular lesion. Analgesics however are indicated for periapical conditions and pain due to pulpitis12. But in this study greatest number of antibiotic prescriptions was written for acute apical abscess
$(62.8 \%)$ and patient with fever and malaise $(62.7 \%)$. Overall, $11.6 \%$ of respondents always prescribed antibiotics after root canal therapy. For chronic periapical lesions and chronic periapical abscess plus sinus tracts, $58.1 \%$ and $55.8 \%$ of respondents prescribed antibiotics respectively which is comparable with study of Nabavezadeh et al where percentage is $58 \%$ and $73.1 \%$ respectively 12.

The drug of choice for these cases was Amoxicillin+clavulanic acid (52.6\%) and Amoxicillin alone $(47.3 \%)$ which is comparable to another study where Amoxicillin $500 \mathrm{mg}$ was the drug of choice (57.6\%) 12.

Prophylactic coverage with antibiotics is indicated only for the highest risk patients who are susceptible to infective endocarditis after bacteremia. The use of prophylactic antibiotics in these patients prevents bloodborne microorganisms from lodging on shunts and prostheses or from multiplying within a depressed system. Patient with HIVIAIDS may be effectively medicated with prophylactic drugs. Whereas in this study $41.8 \%$ of the respondents prescribed antibiotics prophylactically. Patient with prosthetic joint in past 2 years, congenital heart diseases (AV shunt and cardiac valve replacement), mitral valve prolapse, and uncontrolled diabetes are at high risk for development of infections during endodontic treatment and therefore require prophylactic antibiotics 1 .

According to the present study, students and house officers in Multan medical and dental college use antibiotics inappropriately which could lead to problems such as drug resistance, resistant microorganisms and other side effects. It would appear from this study that knowledge about the use of antibiotics is far from ideal. In this study house officers have slightly higher knowledge as compare to the fourth year students. Rational prescribing based on a thorough evidencebased knowledge is essential. The publication of prescribing guidelines and protocols could help to achieve this. Moreover correct educational intervention may also be effective. The use of clinical audit and computers along with other tools to increase knowledge of antibiotic prescribing and improve patient care should not be underestimated.

\section{Conclusion}

This study supports the conclusion that there is a lack of knowledge about the correct indication, type, and dosage of antibiotics in dental practice. Efforts should be made to improve undergraduate education and other educational initiatives on antibiotic prescription skills. The curriculum should provide more focus on prescribing, and students should be taught good prescription practices in the classroom, using hypothetical or real cases.

\section{References}

1. Hargraeves KM, Cohen S, Burmen LH. Cohen's Pathways of the Pulp 10thedition. Elsevier.2011

2. Patel R: Clinical impact of vancomycin-resistant 
enterococci.J Antimicrob Chemother 51(Suppl 3):13, 2003.

3. Tendolkar PM, Baghdayan AS, Shankar N: Pathogenicenterococci: new developments in the 21st century. Cell Mol Life Sci 60:2622, 2003.

4. Vianna ME, Horz HP, Gomes BP, Conrads G: In vivo evaluation of microbial reduction after chemo-mechanical preparation of human root canals containing necrotic pulp tissue. Int Endod $\mathrm{J}(2006)$, 39:484-492.

5. Hecht DW: Prevalence of antibiotic resistance in anaerobic bacteria: worrisome developments. Clin Infect Dis(2004) 39:92.

6. Lee $\mathrm{BH}$. Minimizing prescription writing errors: Computerized prescription order entry. John Hopkins Medical Institutions 2006;1-10.

7. Velo GP. Medication errors: Prescribing faults and prescription errors. Br J Clin Pharmacol 2009;67(6):624-28.

8. Ross S, Loke KY. Do educational interventions improve prescribing by medical students and junior doctors? A systematic review. $\mathrm{Br} \mathrm{J}$ Clin Pharmacol 2009;67(6):662-70.

9. Calligaris L, Panzera A, Arnold L, et al. Errors and omissions in hospital prescriptions: A survey of prescription writing in a hospital. BMC Clin Pharm 2009;9(9):1-6.

10. Harding S, Nicky B, David B. The performance of junior doctors in applying clinical pharmacology knowledge and prescribing skills to standardized clinical cases. $\mathrm{Br} \mathrm{J}$ Clin Phar 2010;69(6):598-606.

11. Ross $\mathrm{S}$, Bond $\mathrm{C}$, Rothnie $\mathrm{H}$, et al. What is the scale of prescribing errors committed by junior doctors? A systematic review. Br J Clin Phar 2008;67(6):629-40.

12. Nabavizadeh MR, Sahebi S, Nadian I. Antibiotic Prescription for Endodontic Treatment: General Dentist Knowledge + Practice in Shiraz. Iran Endod J 2011;6(2):54-59

13. Palmer NA, Dailey YM, Martin MV. Can audit improve antibiotic prescribing in general dental practice? Br Dent J 2001;191:253-5.

14. Walton R: Endodontic Emergencies and Therapeutics. In: Torabinejad M, Walton R, editors. Endodontics principles and Practice, 4th Edition. St. louis: CV Saunders, 2009:153-4. 